\title{
ESTIMATIVA E TÉCNICAS DE AMOSTRAGEM DAS ROÇAS DE MANDIOCA TRADICIONAIS INDÍGENA
}

Bruno Vinícius Castro Guimarães ${ }^{1}$, Ignacio Aspiazú ${ }^{2}$, Abner José de Carvalho $^{3}$, Sérgio Luiz Rodrigues Donato ${ }^{4}$, Laudiceio Viana Matos ${ }^{5}$

1 Professor Doutorando do Instituto Federal do Amazonas Campus São Gabriel da Cachoeira (bvinicius20@yahoo.com.br).

23 Professor Doutor da Universidade Estadual de Montes Claros - UNIMONTES 4 Professor Doutor do Instituto Federal Baiano Campus Guanambi, BA 5 Engenheiro Agrônomo, INCRA, BA/Doutorando na UNIMONTES.

Recebido em: 03/10/2016 - Aprovado em: 21/11/2016 - Publicado em: 05/12/2016 DOI: 10.18677/EnciBio 2016B 048

As roças indígenas são unidades de agricultura de corte e queima de populações tradicionais na Amazônia, geralmente associadas às florestas. A ampla variabilidade da mandioca está relacionada a diversos mecanismos de adaptação, evolução, relação intercultural, dentre outros fatores que contribuem para agrodiversidade. Dessa forma, as técnicas e estimativas das etnovariedades cultivadas são importantes para diversas aplicações, com destaque para os programas de melhoramento genético e mapeamento etnovarietal. Como os campos de cultivo são distribuídos irregularmente, os levantamentos agrícolas tornam-se operações complexas e importantes para 0 conhecimento das variedades cultivadas. Assim, objetivou-se com o presente trabalho realizar e avaliar os procedimentos de estimativa e técnicas de amostragem das roças de mandioca tradicional indígena da etnia Hupd'äh. Utilizaram-se para estimativa das etnovariedades cultivadas nas roças de mandioca quatro levantamentos qualiquantitativos: entrevistas, amostragens por parcelas, transectos linear e diagonal. A diversidade varietal é visível em quaisquer das roças visitadas com fortes evidências dos mecanismos geradores de diversidade. O levantamento das roças de mandioca possibilitou identificar 20, 24, 13 e 17 etnovariedades, respectivamente, nos modelos de entrevistas, parcelas, transectos linear e diagonal. Contudo, o número total de etnovariedades de mandioca identificadas nas roças indígenas foi 32, o que sugere ser a metodologia de amostragem por parcelas a mais representativa do cenário das variedades indígenas.

PALAVRAS-CHAVE: Amazônia, agricultura tradicional, etnovariedades

\section{ESTIMATE AND SAMPLING TECHNIQUES OF TRADITIONAL INDIGENOUS CASSAVA FIELDS}

\footnotetext{
ABSTRACT

Indigenous fields are traditional agricultural units for cutting and burning of populations in the Amazon, usually associated with forests. The wide variability of cassava is related to different mechanisms of adaptation, evolution, intercultural relationship, among other factors that contribute to agro-biodiversity. Thus, the techniques and estimates of cultivated landraces are important for many applications, ENCICLOPÉDIA BIOSFERA, Centro Científico Conhecer - Goiânia, v.13 n.24; p.529 2016
} 
especially for breeding programs and landrace mapping. As the crop fields are unevenly distributed, agricultural surveys become complex, as well as important, to the knowledge of the cultivated varieties. Thus, the aim of the present work was to implement and evaluate the estimation procedures and sampling techniques of the indigenous traditional cassava fields of the Hupd'äh ethnicity. To estimate the landraces cultivated in the four cassava plantations, we used qualitative and quantitative surveys: interviews, sampling plots and linear and diagonal transects. Varietal diversity is visible in any of the visited fields, with strong evidence of the mechanisms that generate diversity. The survey of the cassava fields enabled to identify 20, 24, 13 and 17 landraces, respectively, in the models of interviews, sampling plots, linear and diagonal transects. However, the total number of cassava landraces identified in indigenous fields was 32 , which suggests that the sampling plots methodology is the most representative of the indigenous varieties scenario.

KEYWORDS: Amazon, traditional agriculture, landraces

\section{INTRODUÇÃO}

A mandiocultura é estreitamente associada com o campesinato brasileiro, está presente desde sua gênese e ainda hoje é parte obrigatória da alimentação de vários segmentos de comunidades tradicionais brasileiras das áreas rurais e mais recentemente, as urbanas com a popularização da tapioca ou beiju, em todo o Brasil. Atualmente, maior parte da produção do tubérculo provém de áreas econômica e ecologicamente marginais, cultivada por meio de práticas agrícolas tradicionais, denominadas de agricultura de corte-e-queima (FRASER et al., 2012; SILVA \& MURRIETA, 2014).

$\mathrm{Na}$ floresta amazônica, a mandioca é cultivada em sistema diferenciado de produção com a utilização de pequenas áreas, culturas intercalares e sistema de pousio vegetativo, ao contrário de outras regiões do país que predominam as práticas de monocultura (FREITAS et al., 2011). Essas diferenças no cultivo e uso da mandioca favorecem os programas de melhoramento genético, devido as características agronômicas selecionadas nos sistemas tradicionais de produção. Assim, pode-se assegurar que a Amazônia delimita grande base de variabilidade genética de mandioca com descritores agronômicos importantes tanto para a produção agroecológica (SILVA \& MURRIETA, 2014), quanto para a produção convencional.

FARALDO et al. (2000) observaram que a ampla variabilidade genética de mandioca nas roças indígenas expressa particularidades agronômicas adequadas para a pesquisa científica, tendo como base a conservação varietal in situ. Assim, as etnovariedades de mandioca compõem uma base genética a ser explorada pelas instituições de pesquisa que visam a incorporação de variáveis qualitativas de diversos aspectos na mandiocultura. Além disso, CARRARO et al. (2014) e DIAS \& PENA (2015) acrescentam que as roças tradicionais são apropriadas para a manutenção e desenvolvimento da agricultura sustentável.

No sistema tradicional de produção de mandioca, tanto a sistematização do plantio quanto 0 emprego das variedades atendem aspectos amplamente diversificados. Outra característica dessas roças, além da heterogeneidade de espécies é a diversidade varietal intraespecífica (KAWA et al., 2013).

A mandioca ocupa a maior área plantada das terras agricultáveis da América do Sul. De acordo com relatos históricos, essa cultura predominava no cardápio energético dessas populações, por isso sempre foi associada à ampla diversidade varietal como fonte alimentar. A diversidade de variedades de mandioca em tribos 
indígenas é apresentada em restritos estudos antropológicos (CARNEIRO, 1986; CHERNELA, 1986).

A ampla variabilidade da mandioca está relacionada a diversos mecanismos de adaptação, evolução, relação intercultural, dentre outros fatores que contribuem para agrodiversidade das roças tradicionais (EMPERAIRE \& ELOY, 2015; EMPERAIRE \& GARCÉS, 2016). Assim, as técnicas e estimativas das etnovariedades cultivadas são importantes para diversas aplicações. Como os campos de cultivo são distribuídos irregularmente, os levantamentos agrícolas tornam-se operações complexas e importantes para o conhecimento das variedades cultivadas e de adaptabilidade à realidade agroambiental dessas comunidades indígenas.

Dentro desse contexto, objetivou-se com o presente trabalho realizar e avaliar os procedimentos de estimativa e técnicas de amostragem das roças de mandioca tradicional indígena da etnia Hupd'äh.

\section{MATERIAL E MÉTODOS}

O trabalho foi desenvolvido entre os indígenas da etnia Hupd'äh, nas comunidades: Santa Cruz, Nova Esperança, Nova Fundação, Taracuá Igarapé e Barreira Alta, situadas no extremo noroeste do Amazonas, com as seguintes coordenadas geográficas: Latitude N 0014'46", Longitude W 6924'13" e altitude de $118 \mathrm{~m}$. A precipitação real efetiva no período estudado foi de $3.100,69 \mathrm{~mm}$ ano-1 (GUIMARÂES et al., 2015a) e temperatura média anual de $25^{\circ} \mathrm{C}$.

O procedimento metodológico para estimativa das etnovariedades cultivadas nas roças de mandioca foi composto por quatro levantamentos quali-quantitativos: entrevistas, amostragens por parcelas, transectos linear e diagonal.

Foram realizadas 40 entrevistas com visitas in loco com os integrantes das comunidades indígenas, sobretudo com as mulheres conhecedoras das etnovariedades. O sistema de amostragem foi realizado em 25 roças indígenas, sendo cinco levantamentos por área de produção de mandioca. O procedimento foi executado por dois técnicos e por mulheres conhecedoras das etnovariedades da comunidade local, conforme métodos a seguir:

1 Sistema de amostragem por parcelas quadráticas. Nesse arranjo foram distribuídas quatro parcelas de $4 \mathrm{~m}^{2}$ de modo aleatório nas roças indígenas, perfazendo um total de $16 \mathrm{~m}^{2}$. As parcelas, com dimensões de $2 \times 2 \mathrm{~m}$ foram delimitadas por quatro hastes de madeira nas extremidades e um barbante branco (Figura 1).
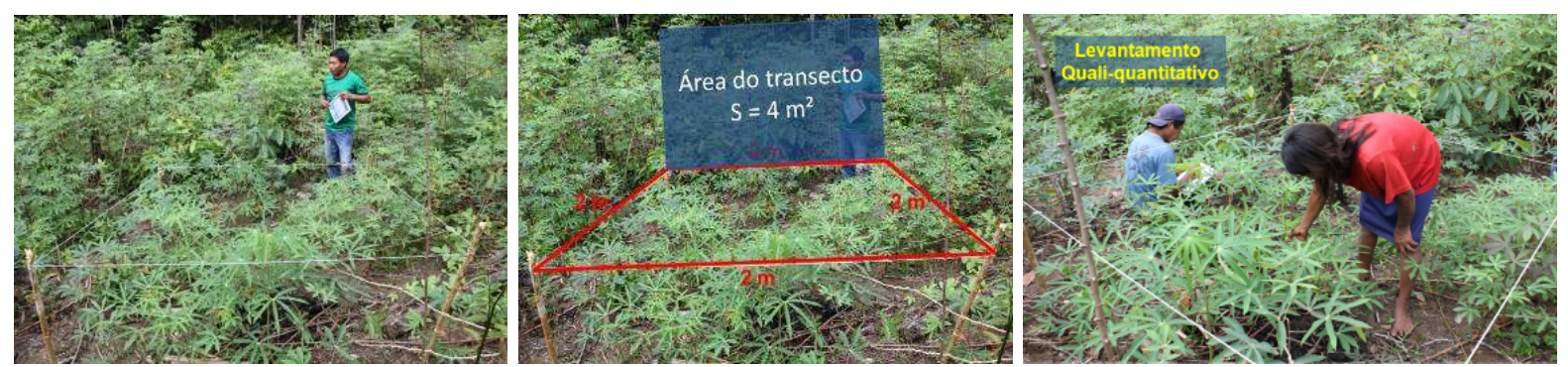

FIGURA 1 Sistema de amostragem das etnovariedades por parcelas

Foto: Bruno Guimarães, (2011). 
2 Sistema de amostragem por transecto linear. Nesse sistema utilizou-se uma trena com $16 \mathrm{~m}$ de comprimento e um bastão com $1 \mathrm{~m}$ de comprimento posicionado de forma perpendicular à trena, proporcionando, dessa forma, uma faixa de amostragem de $16 \mathrm{~m}^{2}$ (Figura 2).
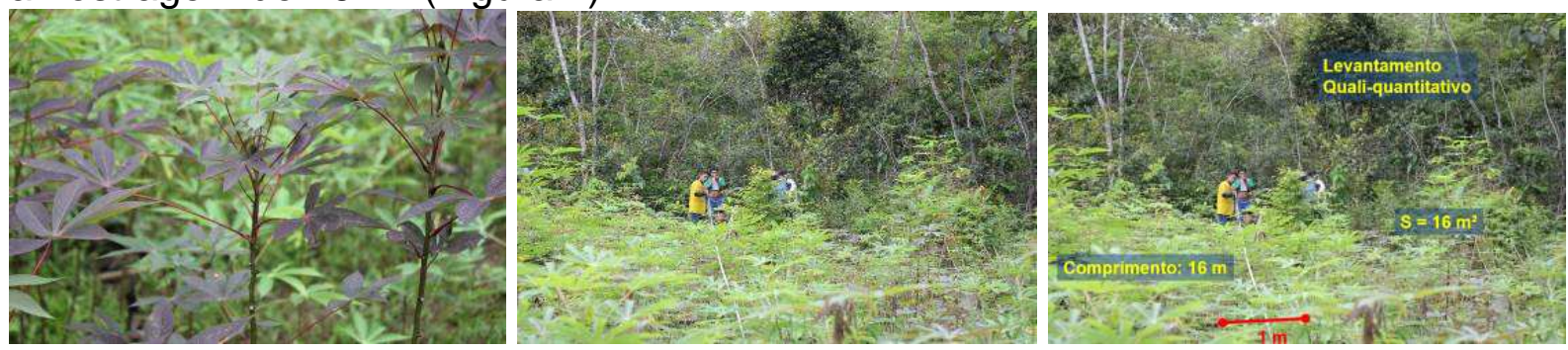

FIGURA 2 Sistema de amostragem das etnovariedades por transecto linear

Foto: Bruno Guimarães, (2011).

3 Sistema de amostragem por transecto diagonal. Nessa amostragem, considerou-se a diagonal principal da roça, e, também com uma trena de $16 \mathrm{~m}$ de comprimento e um bastão com $1 \mathrm{~m}$ de largura disposto perpendicular à trena, delimitou-se uma área de amostragem de $16 \mathrm{~m}^{2}$ (Figura 3).
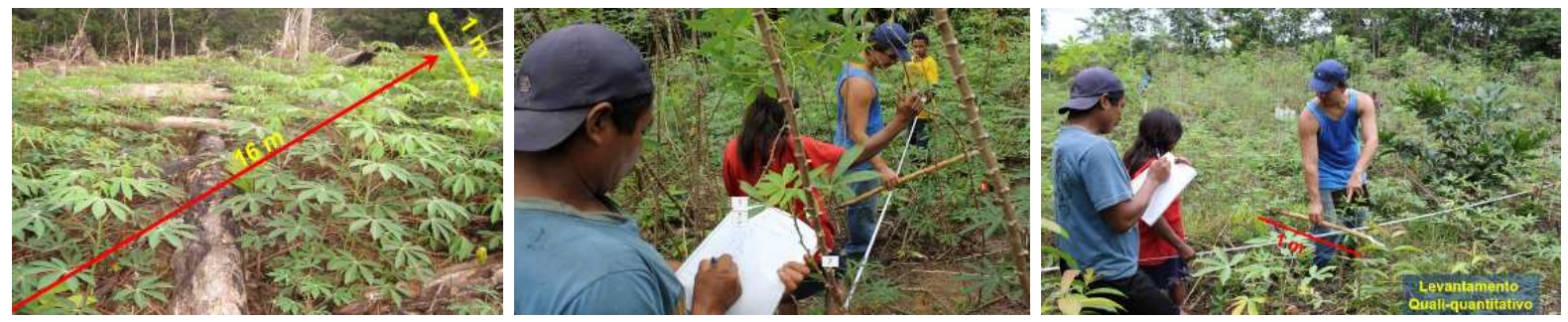

FIGURA 3 Sistema de amostragem das etnovariedades por transecto diagonal Foto: Bruno Guimarães, (2011).

\section{RESULTADOS E DISCUSSÃO}

Por meio da entrevista e do uso dos diferentes sistemas de amostragem: transecto por parcelas, linear e diagonal, foi possível analisar e comparar as técnicas de amostragens com os depoimentos sobre as etnovariedades existentes nas roças de mandioca.

A roça indígena é considerada como um núcleo de produção de alimentos de ampla diversidade cultural (GUIMARÃES, 2014; EMPERAIRE \& GARCÉS, 2016), onde os eventos microevolutivos ocorrem naturalmente. Assim, a arquitetura agrícola é composta por três unidades de produção: a roça, a cultural e a geográfica (Figura 4). Neste sentido, inúmeros estudos de amostragens foram realizados de modo que a comparação entre diferentes métodos pudesse ser usada para testar a existência de um padrão comum ou mais eficiente na representação das etnovariedades locais (CARNEIRO, 1986; CHERNELA, 1986).
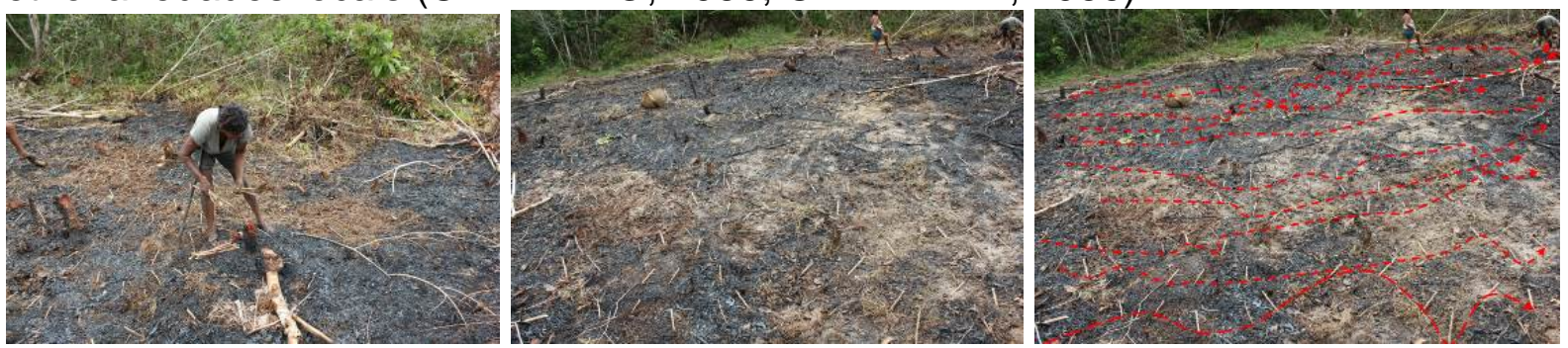

FIGURA 4 Sistematização e arranjo das manivas numa roça de vegetação secundária recém queimada

Foto: Bruno Guimarães, (2011). 
Foram identificadas 32 etnovariedades de mandioca nas comunidades estudadas (Figura 5). MARTINS (2005) afirma que a ampla diversidade de mandioca apresenta forte relação com o manejo tradicional das etnovariedades. Após a supressão vegetal, primária ou secundária, ocorre a implantação da cultura da mandioca, com isso se estabelecem uma população definida pelos marcos genéticos e demográficos. São definidos a composição e a densidade da população, número e tipo de variedades e a disposição espacial desses indivíduos no cenário agroflorestal. No entanto, a retirada da mata ou a perturbação antrópica do ecossistema natural favorece a pressão de seleção das espécies espontâneas relacionadas ou não à cultura agrícola. Isso reforça a teoria de que pelo menos algumas espécies tenham sido domesticadas de modo simultâneo com a mandioca, processo marcado pela habilidade de combinação ecológica dessas espécies.

A entrevista com as mulheres agricultoras possibilitou o levantamento qualitativo de 20 etnovariedades, sendo que as variedades $\mathrm{H} \rightarrow \mathrm{w}^{\prime}$ tig (maniva cigarra) e Nèg sak tig (maniva cera de mel) respondem por $25 \%$ das etnovariedades cultivadas nas roças indígenas (Figura 5). A predominância dessas variedades nas roças deve-se a ampla utilidade das raízes na fabricação de goma, beiju e caxiri, esse último refere-se a uma bebida fermentada da polpa. As variedades são classificadas como precoce, com o intervalo do plantio à colheita entre 6 a 12 meses.

As roças de mandioca são amplamente diversificadas nos aspectos morfológicos, bioquímicos e genéticos (OLIVEIRA et al., 2012). A população indígena, predominantemente, as mulheres, apresenta alto grau de percepção para selecionar, identificar e cultivar as variedades, processo definido como domesticação. Os atributos morfológicos utilizados na classificação das etnovariedades são relacionados aos descritores da planta, vegetativo e radicular, no primeiro, destaca-se na identificação das variedades, o sistema foliar, a forma, dimensão e coloração dos pecíolos, folíolos e ramos. Nas raízes comerciais, observa-se, o tamanho e coloração, como a polpa branca, creme, amarela, rica em caroteno ou vermelha com a predominância do licopeno. Assim, a preferência pela variedade e o sistema de cultivo são objeto de adaptabilidade e seleção cultural pelos integrantes das comunidades tradicionais (MATOS et al., 2014).

A percepção morfológica das variedades de mandioca pelas agricultoras indígenas é fortemente desenvolvida e conectada com a prática, como ressalta uma comparação entre o sistema classificatório dos indígenas do Rio Negro e a análise morfométrica multivariada de 32 caracteres agronômicos (MARTINS, 2005). Neste estudo, observou-se estreita concordância entre os dois sistemas, conforme argumenta MARTINS (2005). OLIVEIRA et al. (2012) acrescenta que os marcadores microssatélites são ferramentas eficientes para identificar novos clones de mandioca, que compõe a tão diversificada roça tradicional de mandioca.

Ainda de forma semelhante, MARTINS (2005) aprofundou os estudos por meio de marcadores isoenzimáticos e genéticos - RAPD (Random Amplified Polimorphic DNA) e RFLP (Restriction Fragment Length Polymorphism) e evidenciou ampla eficiência entre os métodos supracitados. Dos caracteres avaliados (32), oito foram relacionados a $80 \%$ da variação encontrada, sendo a maioria dos atributos estudados (seis) associados às raízes. Disso poder-se-ia assegurar que o sistema caboclo de classificação fosse fortemente baseado nos aspectos do sistema radicular. Diferentemente, os indígenas utilizam predominantemente descritores da parte aérea, e estes apresentam correlação nula com os atributos das raízes 
comerciais, uma divergência que carece de bases científicas para completa elucidação.

O levantamento etnovarietal por meio de parcelas quadráticas identificou 24 variedades, sendo que a mandioca D'öp tig (maniva japu) representa $12,5 \%$ da preferência entre os indígenas (Figura 5). Essa etnovariedade apresenta ciclo de nove meses e polpa de coloração vermelha, rica em licopeno e grande aptidão para fabricação de farinha.

A diversidade varietal é visível em quaisquer das roças visitadas e as evidências dos mecanismos geradores de diversidade são expressivas. Fato também evidenciado por GUIMARÃES et al. (2015b) com a mesma etnia. O método amostral por meio do transecto linear identificou 13 etnovariedades, sendo esse sistema de amostragem o mais limitado, tanto em termos quantitativos, quanto qualitativos (Figura 5). O sistema de amostragem por meio do transecto diagonal identificou 17 etnovariedades, que correspondem às variedades $\mathrm{H}$ (w' tig, K'ók k' óh tig, Neg sák tig, Sã rã), e representam 52,94\% dos exemplares amostrados (Figura $5)$.

A diversidade genética de mandioca está ancorada em diversos fatores relacionados com a adaptação e evolução da cultura. MARTINS (2005) argumenta que a existência diversificada das variedades de mandioca na roça, permite cruzamentos inter e intraespecíficos, ampliando, dessa forma, a recombinação genética. Além disso, a permuta de variedades dentro e entre as comunidades indígenas favorece a propagação, multiplicação e distribuição espacial das etnovariedades, como relatado em diversos estudos (CARNEIRO, 1986; CHERNELA, 1986; CLEMENT, 1999; EMPERAIRE \& ELOY, 2015).

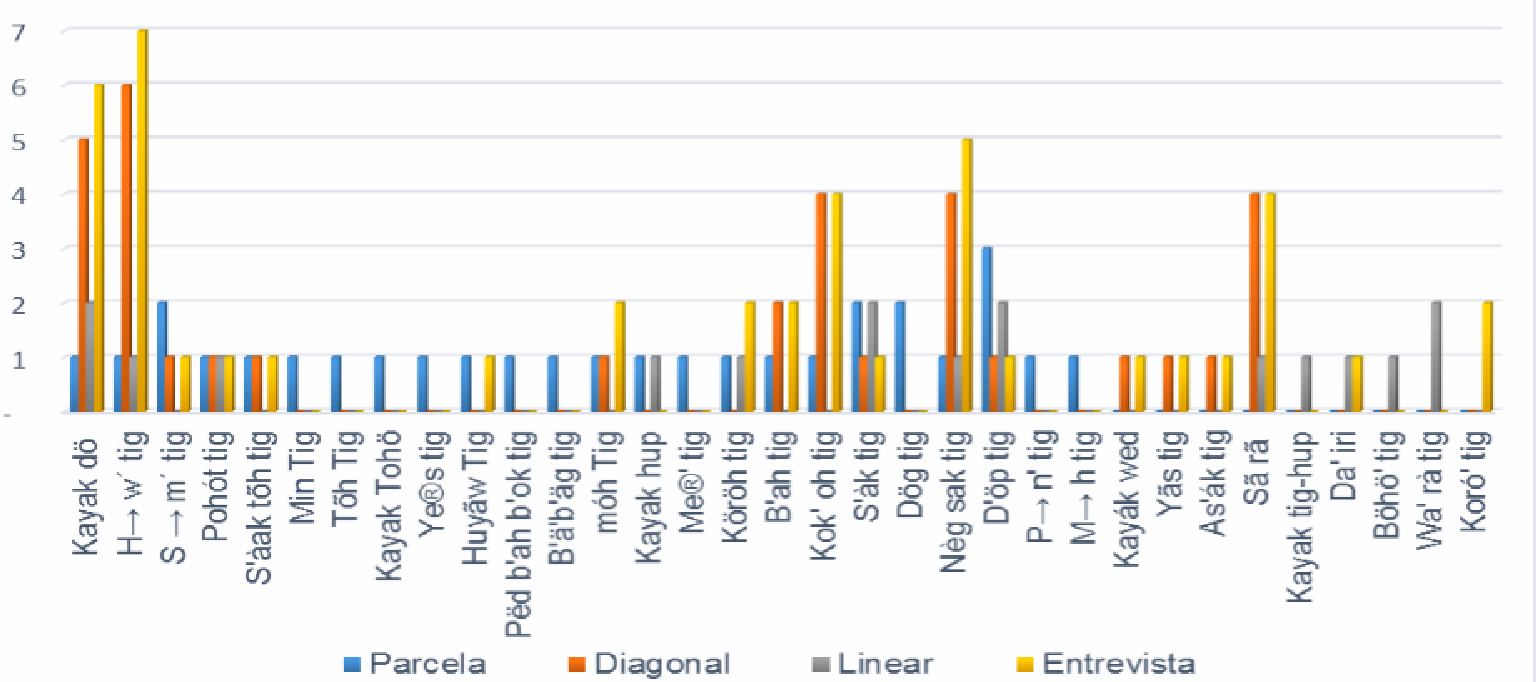

FIGURA 5 Comparação entres os métodos de amostragens da mandiocultura tradicional indígena, Rio Tiquié, Amazonas, 2011.

\section{CONCLUSÃO}

A agricultura indígena tradicional gera e amplifica a diversidade genética das roças cultivadas em um processo contínuo. O estudo das roças de mandioca possibilitou identificar 20, 24, 13 e 17 etnovariedades, respectivamente, nos modelos de entrevistas, parcelas, transecto linear e diagonal. Contudo, o número total de variedades de mandioca identificadas nas roças indígenas foi 32, o que sugere ser a metodologia de amostragem por parcelas a mais representativa do conjunto das variedades indígenas. 
A conservação in situ das variedades de mandioca é extremamente importante para o desenvolvimento da agricultura em todos os níveis de tecnologia e para o melhoramento genético clássico ou biotecnológico.

\section{AGRADECIMENTOS}

Aos Institutos Federais: IFAM e IF-Baiano.

À Universidade Estadual de Montes Claros - UNIMONTES.

\section{REFERÊNCIAS}

CARRARO, J. L; POCAI, L. H; PEIXER, Z. I. O Povo que Caminha sob o Sol: A Experiência do Projeto Carbono Social em Rede no Território Indígena LaklãNõ. Cadernos de Agroecologia - ISSN 2236-7934, v.9, n.4, Nov 2014. Disponível em: <http://aba-agroecologia.org.br/revistas/index.php/cad/article/view/16804/10633>.

CLEMENT, C.R. 1492 and the loss of Amazonian crop genetic resources.l. The relation between domestication and population decline. Economic Botany, 53: 188-202. 1999. Disponível em: < https://www.jstor.org/stable/4256179?seq=1\#page_scan_tab_contents $>$. (doi) AND iid:(10.2307/i389495).

CHERNELLA, J. M. "Os cultivares de mandioca na área do Uaupés (Tukâno)". Em RIBEIRO, D. (ed.). Suma Etnológica brasileira: 1. Etnobiologia. Petrópolis, Vozes, pp. 151-158, 1986. Disponível em: < http://www.scielo.br/scielo.php?script=sci_nlinks\&ref=000083\&pid=S01034014200500010001300006\&lng=en>.

DIAS, J. B; PENA, L. L. S. AS PRÁTICAS/SABERES AGRÍCOLAS, CONTRIBUIÇÃO À CONSERVAÇÃO DA BIODIVERSIDADE LOCAL? O CASO DE ANDRÉ DO MATO DENTRO, SANTA BÁRBARA, MG. Geosaberes, Fortaleza, v. 6 , número especial (2), p. 90 - 102, Novembro. 2015. Disponível em: < http://www.geosaberes.ufc.br/seer/index.php/geosaberes/article/view/414/379>.

ISSN: 2178-0463.

EMPERAIRE, L.; ELOY, L. Amerindian Agriculture in an Urbanising Amazonia (Rio Negro, Brazil). Bulletin of Latin American Research, Vol. 34, No. 1, pp. 70-84, $2015 . \quad$ Disponível em:

http://onlinelibrary.wiley.com/doi/10.1111/blar.12176/epdf?r3_referer=wol\&tracking_a ction=preview_click\&show_checkout $=1$ \&purchase_referrer $=$ www.google.com.br\&pur chase_site_license=LICENSE_DENIED>. DOI: 10.1111/blar.12176.

EMPERAIRE, L.; GARCÉS, C. L. L. DOSSIÊ DINÂMICAS DAS AGRICULTURAS AMAZÔNICAS. Dinâmicas das agriculturas amazônicas. Bol. Mus. Para. Emílio Goeldi. Ciênc. hum. vl.11 no.1 Belém jan./abr. 2016. Disponível em: < http://www.scielo.br/scielo.php?script=sci_arttext\&pid=S1981-

$81222016000100013 \&$ Ing $=$ pt\&nrm=iso\&tlng $=$ en $>$.

DOI:

http://dx.doi.org/10.1590/1981.81222016000100002.

ESUMA, W.; RUBAIHAYO, P.; PARIYO, A.; KAWUKI, R.; WANJALA, B.; NZUKI, I.; HARVEY, J. J. W.; BAGUMA, Y. Genetic diversity of provitamnin A cassava in Uganda. Journal of Plant Studies, Canadá, v. 1, n. 1, p. 60-71, 2012. Disponível 
em: <http://www.ccsenet.org/journal/index.php/jps/article/view/13540/10307>. DOI: http://dx.doi.org/10.5539/jps.v1n1p60.

FARALDO, M. I. F.; SILVA, R. M.; ANDO, A.; MARTINS, P. S. Variabilidade genética de etnovariedades de mandioca em regiões geográficas do Brasil. Scientia Agricola, v.57, n.3, p.499-505, jul./set. 2000. Disponível em: < http://www.scielo.br/pdf/sa/v57n3/2682.pdf>. $\quad$ DOI: $10.1590 / S 0103-$ 90162000000300020.

FRASER, J. A.; ALVES-PEREIRA, A.; JUNQUEIRA, A. B.; PERONI, N.; CLEMENT, C. R. (2012) Convergent Adaptations: Bitter Manioc Cultivation Systems in Fertile Anthropogenic Dark Earths and Floodplain Soils in Central Amazonia. PLoS ONE 7(8): e43636. Disponível em: <http://journals.plos.org/plosone/article?id=10.1371/journal.pone.00436366> Doi:10.1371/journal.pone.0043636.

FREITAS, C. G.; FARIAS, C. S.; VILPOUX, O. F. A produção camponesa de farinha de mandioca na Amazônia sul ocidental. Boletim Goiano de Geografia, v. 31, n. 2, p. 29-42, 2011.2 Disponível em: <http://www.redalyc.org/pdf/3371/337127156002.pdf>. 10.5216/bgg.V31i2.16843.

GUIMARÃES, B. V. C.; SOUZA, E. B., ABREU, A. M. A., DONATO, S. L. R., ASPIAZU, I. Captação e aproveitamento da água da chuva para fins não potáveis e potáveis. Enciclopédia Biosfera., v.11, p.2926 - 2939, 2015a. Disponível em: < http://www.conhecer.org.br/enciclop/2015b/multidisciplinar/Captacao\%20e\%20Aprov eitamento.pdf>.

GUIMARÃES, B. V. C.; SOUZA, E. B.; MIRANDA, M. J. S.; ABREU, A. M. A.; GUIMARÃES, M. M. C. Levantamento quali-quantitativo sobre variedades de mandioca da etnia Hupd'äh no noroeste do amazonas. Enciclopédia Biosfera, Centro Científico Conhecer - Goiânia, v.11 n.22; p. 2119 2015b. Disponível em: <http://www.conhecer.org.br/enciclop/2015c/agrarias/levantamento\%20quali.pdf>.

DOI: http://dx.doi.org/10.18677/Enciclopedia_Biosfera_2015_185.

GUIMARÃES, F. A. M. POVOS INDÍGENAS NO BRASIL E AS LIÇÕES DA FLORESTA CULTURAL: A revolução da cultura da mandioca na economia do atlântico sul e no continente africano. Revista do Programa de Pós-Graduação em Crítica Cultural. Pontos de Interrogação, v. 4, n. 2, jul./dez. 2014. Disponível em: < http://www.revistas.uneb.br/index.php/pontosdeint/article/view/1680/1113 >.

MARTINS, P. S. Dinâmica evolutiva em roças de caboclos amazônicos, ESTUDOS AVANÇADOS 19 (53), 2005. Disponível em: < http://www.scielo.br/scielo.php?script=sci_arttext\&pid=S0103-40142005000100013>. DOI: http://dx.doi.org/10.1590/S0103-40142005000100013.

MATOS, L. M.; KER, J. C.; CARDOSO, I. M.; LANI, J. L.; SCHAEFER, C. E. G. R. O conhecimento local e a etnopedologia no estudo dos agroecossistemas da Comunidade Quilombola de Brejo dos Crioulos. Soc. \& Nat., Uberlândia, 26 (3): 
497-510.

2014.

Disponível

em:

$<$ http://www.seer.ufu.br/index.php/sociedadenatureza/article/view/23773>

DOI:

http://dx.doi.org/10.1590/1982-451320140308>.

OLIVEIRA, M. V. C.; BALIZA, D. P.; SOUZA, G. A.; CARVALHO, S. P.; ASSIS, L. H. $B$. Caracterização de clones de mandioca utilizando marcadores microssatélites. Revista Ciência Agronômica, v. 43, n. 1, p. 170-176, jan-mar, 2012. Disponível em: < http://www.ccarevista.ufc.br/seer/index.php/ccarevista/article/view/1136/665>. ISSN 1806-6690.

KAWA, N. C.; MCCARTY, C.; CLEMENT, C. R. Manioc Varietal Diversity, Social Networks, and Distribution Constraints in Rural Amazonia. Current Anthropology, v. 54, p. 764-770, 2013. Disponível em < http://www.journals.uchicago.edu/doi/10.1086/673528> DOI: 10.1086/673528.

SILVA, H. A \& MURRIETA, R. S. S. Mandioca, a rainha do Brasil? Ascensão e queda da Manihot esculenta no estado de São Paulo. Bol. Mus. Para. Emílio Goeldi. Cienc. Hum., Belém, v. 9, n. 1, p. 37-60, jan.-abr. 2014. Disponível em: <www.scielo.br/pdf/bgoeldi/v9n1/04.pdf>. Doi: 10.1080/10934529.2013.81550. 